\title{
A Survey on Quantitative Assessment of the Quality of Tone-Mapped Images using TMQI- LI
}

\author{
K C Asha \\ Sree Buddha College Of Engineering \\ Prof:Anil A R, \\ Sree Buddha College Of Engineering
}

\begin{abstract}
Even though the knowledge of tone mapping is continuously developing, many unanswered questions and hypotheses still remain to be solved. On that account, we are quite far away from having an accurate computational model for the compression of high dynamic range images to low dynamic ones. It is; therefore; of particular importance and necessity to conduct experimental subjective evaluations of the computational approaches that incorporate various tone mapping operators to judge and validate them properly. Moreover, the evaluations give us a deeper insight into the inquired area and, besides the evaluation of existing methods they can lead to proposals of new ones. The goal of Image Quality Assessment (IQA) is to computationally predict human perceptions of image quality. In order to improve the quality of the image two factors are considered, they are structural fidelity and statistical naturalness. Then the proposed iterative algorithms are used, so as to improve the quality of the image. Numerical and subjective experiments make evident that the proposed algorithm consistently produces better quality tone mapped images even when the initial images of the iteration are created by the most competitive TMOs.
\end{abstract}

Key Words: Tone mapping operators, Noise estimation, Accuracy, Human Visual system and Quality assessment.

\section{INTRODUCTION}

The luminance of a natural scene often has a high dynamic range (HDR), varying between $10^{3}$ to $10^{5} \mathrm{~cd} / \mathrm{m}^{2}$. However, a normal digital display only has a low dynamic range (LDR) about $10^{2} \mathrm{~cd} / \mathrm{m}^{2}$.Tone mapping operators (TMO) fill in the gap between HDR imaging and visualizing these images on standard displays by compressing the dynamic range of HDR images [2]. TMOs provide a nice stand-in for HDR display technology, which is currently immature and expensive. Due to the existence of both light and dark areas in the same scene, even the best exposure shots fail to capture the detail and color appearance of the sky in the background and the bricks in the foreground. By contrast, the acquaintance of both the indoor and the outdoor areas has been greatly improved in tone mapped images. On the other hand, printed media are typically not HDR. Nevertheless of how fast HDR display technologies penetrate, there will be a strong need to prepare HDR imagery for display on LDR devices [1]. In addition, reducing the dynamic range of an HDR image while preserving its structural detail and natural appearance is by itself a problem for human and computer vision study. In recent years, many TMOs have been recommended. Most of them were demonstrated on special examples without being thoroughly tested on well-established and subject validated image quality assessment (IQA) models. Subjective evaluation is a straightforward and useful quality measure, because the human visual system is the ultimate receiver in most applications. However it is expensive, time consuming, and perhaps most importantly, can hardly guide automatic optimization algorithms. In the context of subjective quality assessment of tone mapped images, subjects usually directly compare several different tone mapped images without referencing the corresponding HDR images. Therefore, they may be unaware of certain structure detail loss in tone mapped images that may convey important information in HDR images. For this reason, subjective evaluation may not be a golden evaluation criterion. Objective quality assessment of tone mapped images is a thought-provoking problem due to the different dynamic ranges between the referenced image and the tone mapped LDR image. Some attempts have been made for tangibly assessing the quality of HDR images. The HDR visible difference predictor tries to predict the visible difference between two HDR images with the same range.

\section{LITERATURE SURVEY}


HDR rendering is becoming increasingly popular now-a-days and is supported by OpenGL, the de-facto standard for rendering $2 \mathrm{D}$ and $3 \mathrm{D}$ graphics. Instead of clamping the color values in the frame buffer between the values of 0.0 and 1.0 after each fragment shader run, HDR OpenGL rendering allows the colors to be saved in true floating point values outside the default range of 0.0 and 1.0. This leads to the preservation of more detail as well as provides the designer with the ability to configure the lighting of the scene better with more realistic lighting parameters.

Once the radiance map is obtained, either from the multiply exposed images in case of photographs taken with optical images or the floating point rendered color values in computer graphics, it is tone mapped to a lower gamut (8 bit/color/pixel) of the SDR display. These algorithms try to replicate the local-adaptation behaviour of the human visual system. The human eye deals with the vast range of real-world illuminations by changing their sensitivity to be responsive at different illumination levels in a highly localized fashion, enabling us to see the details both in the bright and dark regions. The tone-mapping algorithms compute either a spatially varying transfer function or shrink the image gradients to fit into the available dynamic range.

Tone-mapped FR-IQA metrics employ average pooling that weights all pixels equally. Using dissimilar pooling strategies for combining local quality scores to yield the final quality index of the processed SDR image is wellresearched. Using perceptual pooling methods for quality evaluation of HDR images is less well studied. The authors have proposed Saliency weighted Tone-Mapped Quality Index (STMQI) that employs thoughtfulness based on Information Maximization [3] model to find the salient regions of the image.

The TMQI algorithm is based on the combination of two image quality indicators: 1) a multiscale image fidelity metric based on a modified structural similarity (SSIM) index and 2) a measure based on natural scene statistics (NSS): the mean and standard deviation of pixel intensities. Subsequently the effervescent range of the HDR images is much higher than that of standard SDR displays, the TMOs cannot preserve all the details of the HDR versions; nevertheless it must ensure that the SDR image is structurally similar to the HDR version. The SSIM-inspired component takes into account this aspect of signal fidelity. In addition, the SDR image must also ensure that it looks natural because the human visual system is trained on NSS that appear irrespective of image content. The TMQI algorithm takes into account only the pixel luminances. Both the HDR and SDR images are converted from RGB color space to the Yxy color space and the algorithm is applied only to the Y component.

The TMQI model is used for quantitative assessment of the Quality of tone-mapped images using their accompanying HDR images as references. In [11], it is demonstrated that inclusion of prediction of salient regions of an Image in tone-mapped image quality assessment, results in an improved tone-mapping quality metric. The proposed SMQI shows strong correlation with subjective evaluations of image quality, and accomplishes higher spearman and kendall Rank-order correlation coefficients than that of the standard TMQI metric. Further development of this work is directed at assessing alternative saliency measures and towards more sophisticated measures of naturalness based on both global and local image statistics towards stronger accordance with Human decisions.

A detailed analysis of the multi-scale fusion problem in the context of JPEG splicing forgeries[12]; It clearly shows the fusion of candidate maps obtained on multiple scales of analysis that can improve the tampering localization performance of sliding window-based detectors by combining the benefits of small-scale and large-scale analysis. A novel multi-scale fusion technique based on energy minimization and threshold drift; the latter is a key component that allows exploiting the dependencies between different scales of analysis.

Two novel fusion techniques based on heuristic top-down and bottom-up refinement of an initial single-scale tampering map; the refinement follows simple rules corresponding to the expected dependencies between smallscale and large-scale analysis. Later on, the choice of the analysis windows for multi-scale fusion can be investigated. The preferred number of candidate maps will be determined, and the sliding window overlap that would guarantee good localization and computation performance. The latter will become particularly important when dealing with high-resolution images. The suitability of multi-scale fusion for other forensic features will be investigated. Positive results would indicate feasibility of a combined multi-modal and multi-scale approach.

In this paper [13] a simple perceptual pooling techniques that take into account the local contrast that improve the performance of the TMQI algorithm for quality evaluation and propose a different NSS model to better qualify the image naturalness. In toting to tone-mapping artifacts, the proposed methods show good correlation with human observers for JPEG compressed tone-mapped images.

The main objective of image quality assessment method is to evaluate the quality of tone-mapped images. The novelty of the [14] lies in employing the sparse-domain coefficients to compare the cross dynamic range similarity and evaluate the naturalness. 
Firstly, with a dictionary trained from HDR images, the pristine HDR and tone-mapped LDR images are projected to the unified sparse space to compare the local sparse-domain similarity. Second, a novel naturalness measure is developed based on the features extracted from the coefficients in sparse representation. Experimental results of the proposed method achieve higher correlation with the subjective data. The future work is mainly the optimization of TMO algorithms based on the design philosophy of the proposed SMTI model.

The assumption that there is a single optimal solution to HDR image tone mapping is completely neglected. Tone mapping is a subjective process that is guided by the taste and preferences of the artist and situational contextual considerations. A new method that [15] can learn these preferences from a small set of calibration images and apply them to new images for tone mapping is mainly used here.

Unlike previous work which is focused on LDR image retouching, this approach allows batch processing a large number of HDR images with different styles and therefore can greatly benefit practitioners in film, video, and photography industries.

A simple but effective tone mapping operator is used here [16], which can achieve impressive results with low complexity in both the formulation and computation. The key point of this operator is to calculate a scalar matrix by which the input HDR image can be mapped into a displayable LDR image.

The mainly addressed problems of HDR video compression and tone mapping that are important components of the high dynamic range imaging pipeline are discussed here [17]. In the HDR video part we proposed a new color space which encodes the full luminance range that is visible by the human eye.

The use of this color space for 12-bit MPEG-4 compression leads automatically to HDR video encoding without any changes in the MPEG standard. Another presented solution relies on 8-bit HDR video encoding. To overcome these problems a tone mapping approach is developed, that minimize the contrast distortion with respect to the real world scene perception by the human observer. The same computational framework can be used to maximize local contrast just by changing the optimization condition. The whole algorithm maps well to existing graphics hardware which significantly improves the computation performance.

By providing a lossy,[18][19] high dynamic range image format that is backwards compatible with existing JPEG software, here an important barrier to the adoption of HDR imaging technology by digital camera manufacturers and web content providers can be removed. The sub-band encoding method presented couples a high quality, tonemapped (i.e., output-referred) foreground image with metadata that enables HDR software to recover the original, scene-referred luminance's at 16-bit resolution. Naïve applications see only the tone-mapped version, which still encompasses the larger dynamic range, albeit with 8-bit precision.

\section{CONCLUSIONS}

The modified structural fidelity term results in a more meaningful and useful structural fidelity map and guides all the proposed optimization algorithms to recover fine detail with more robustness to noise artifacts. An image dependent statistical naturalness model and quantify the unnaturalness of tone mapped LDR images based on a subjective experiment is build. eTMQI outperforms TMQI on structural fidelity and statistical naturalness terms separately. An iterative optimization algorithm compare eTMQI and TMQI in the space of all images which also produce better quality tone mapped images when eTMQI is chosen as the optimization goal. Numerical and subjective experiments suggest that eTMQI is a robust objective quality metric for quality prediction of tone mapped images and consistently outperforms TMQI.

\section{REFERENCES}

[1] Hojatollah Yeganeh and Zhou Wang, “Objective assessment of tone mapping algorithms,” proceedings of $2010 \mathrm{IEEE} 17^{\text {th }}$ International conference on image processing

[2] Marcus Barkowsky, Patrick Le Callet, “On the perceptual similarity of realistic looking tone mapped high dynamic range images,” proceedings of 2010 IEEE $17^{\text {th }}$ International conference on image processing

[3] Fabrizio Guerrini, Masahiro Okuda, Nicola Adami, and Riccardo Leonardi, “High Dynamic Range Image Watermarking Robust Against Tone-Mapping Operators” IEEE TRANSACTIONS ON INFORMATION FORENSICS AND SECURITY, VOL. 6, NO. 2, JUNE 2011 
[4] Hojatollah Yeganeh and Zhou Wang,

“Objective assessment of tone mapping algorithms,” proceedings of 2010 IEEE $17^{\text {th }}$ International conference on image processing.

[5] Hamid Reza Nasrinpour University of Manitoba Winnipeg, MB, Canada, "SALIENCY WEIGHTED QUALITY ASSESSMENT OF TONE-MAPPED IMAGES,”978-1-4799-8339-1/15/\$31.00 @2015 IEEE.

[6] B. A. Wandell, Foundations of vision. Sinauer Associates, 1995. 1

[7] E. Reinhard, W. Heidrich, P. Debevec, S. Pattanaik, G. Ward, and K. Myszkowski, High Dynamic Range Imaging: Acquisition, Display, and Image-based Lighting. Morgan Kaufmann, 2010. xiii, 1, 2, 7, 12

[8] E. Reinhard, M. Stark, P. Shirley, and J. Ferwerda, \Photographic tone reproduction for digital images," ACM Transactions on Graphics, vol. 21, no. 3, pp. $267\{276$, 2002. xi, xiii, xiv, xv, xvi, 3, 7, 9, 10, 11, 12, 23, 24, 25, 29, 37, 38, 39, 41, 42, 43, 45, 47, 48

[9] F. Drago, K. Myszkowski, T. Annen, and N. Chiba, \Adaptive logarithmic mapping for displaying high contrast scenes," Computer Graphics Forum, vol. 22, pp. 419\{426, 2003. xiii, xvi, 3, 10, 11, 12, 24, 29, 39, 41, 42, 43

[10] Q. Shan, J. Jia, and M. S. Brown, \Globally optimized linear windowed tone mapping," IEEE Trans. on Visualization and Computer Graphics, vol. 16, no. 4, pp. $663\{675,2010.3,7,10$

[11] M.Cadik and P. Slavik, IThe naturalness of reproduced high dynamic range images," in International Conference on Information Visualisation, pp. 920-925, 2005. 3

[12] F. Drago, W. L. Martens, K. Myszkowski, and H.-P. Seidel, \Perceptual evaluation of tone mapping operators," in ACM SIGGRAPH 2003 Sketches \& Applications, pp. 1\{1, 2003. 3

[13] C. Cavaro-Menard, L. Zhang, and P. Le Callet, \Diagnostic quality assessment of medical images: Challenges and trends," in IEEE 2nd European Workshop on Visual Information Processing, pp. 277\{284, 2010. 3

[14] Z. Wang and A. C. Bovik, \Mean squared error: love it or leave it? a new look at signal delity measures," IEEE Signal Processing Magazine, vol. 26, no. 1, pp. $98\{117,2009$. xiv, 3, 13, 14, 15, 16

[15] Z. Wang, A. C. Bovik, H. R. Sheikh, and E. P. Simoncelli, \Image quality assessment: from error visibility to structural similarity," IEEE Trans. on Image Processing, vol. 13, no. 4, pp. $600\{612,2004.3$, 13, 15, 17

[16] R. Mantiuk, S. J. Daly, K. Myszkowski, and H.-P.Seidel, \Predicting visible di erences in high dynamic range images: model and its calibration," in Proc. SPIE, vol. 5666,pp. 204\{214, 2005. 3

[17] T. O. Aydin, R. Mantiuk, K. Myszkowski, and H.-P. Seidel, IDynamic range independent image quality assessment," ACM Transactions on Graphics, vol. 27, no. 3, p. 69,2008.

[18] H. Yeganeh and Z. Wang, \Objective quality assessment of tone-mapped images,"IEEE Trans. on Image Processing, vol. 22, no. 2, pp. $657\{667,2013 . x i, x i v, 3,10,15,17,18,19,20,21,22,23,24,28,29,30,31,32,39,42$

[19] J. M. DiCarlo and B. A. Wandell, \Rendering high dynamic range images," in Electronic Imaging, pp. 392 \{401, International Society for Optics and Photonics, 2000

[20] G. W. Larson, H. Rushmeier, and C. Piatko, \A visibility matching tone reproduction operator for high dynamic range scenes," IEEE Trans. on Visualization and Computer Graphics, vol. 3, no. 4, pp. 291\{306, 1997. 10 